\title{
Autonomous UAV Simulator for Research and Development Applied to 5G Networks
}

\author{
Carlos Eduardo Dias Vinagre \\ Neto \\ Federal University of Pará \\ Belém, Pará, Brazil \\ carlos.vinagre.neto@itec.ufpa.br
}

\author{
Ailton Pinto de Oliveira \\ Federal University of Pará \\ Belém, Pará, Brazil \\ ailton.pinto@itec.ufpa.br
}

\author{
Felipe Henrique Bastos e \\ Bastos \\ Federal University of Pará \\ Belém, Pará, Brazil \\ felipe.bastos@itec.ufpa.br
}

\author{
Emerson Oliveira Junior \\ Federal University of Pará \\ Belém, Pará, Brazil \\ emersonjr@ufpa.br
}

\author{
Aldebaro Klautau \\ Federal University of Pará \\ Belém, Pará, Brazil \\ aldebaro@ufpa.br
}

\begin{abstract}
Unmanned aerial vehicles (UAVs) are being used in many applications, such as surveillance and product delivery. Currently, many UAVs are controlled by $\mathrm{WiFi}$ or proprietary radio technologies. However, it is envisioned that $5 \mathrm{G}$ and beyond 5G (B5G) networks can connect the UAVs and increase the overall security due to improved control by operators and governments. Soon, UAVs will also be used as mobile radio base stations to extend reach or improve the network capacity. All this motivates intense research on $5 \mathrm{G}$ technologies for supporting UAV-based applications. However, there are currently few simulation tools for testing and investigating telecommunication systems that involve UAV solutions. For instance, modern $5 \mathrm{G}$ networks use multiple antennas that enable beamforming. A realistic simulation, in this case, requires not only support for beamforming but also for realistic UAV trajectories, which impact the communication channel evolution over time. To evaluate scenarios with connected UAVs, this paper presents a tool that simulates flights in a virtual environment, gathers information about the channels among UAVs and the mobile network, and calculates performance indicators regarding the communication system.
\end{abstract}

\section{KEYWORDS}

UAV, unreal engine, vehicular networks, MIMO, 5G

\section{INTRODUCTION}

Unmanned aerial vehicles (UAVs) can be used in a large variety of applications, such as agriculture mapping, surveillance, and many others. Researches show that the number of drones will exceed 7 million in 2020 [3]. This rapid increase in the number of UAVs will have substantial impacts on $5 \mathrm{G}$ wireless systems and beyond $5 \mathrm{G}$ (B5G). Many UAVs are currently controlled by WiFi or proprietary radio technologies. However, this brings several security issues, and it is envisioned that legislation will enforce a drone 5G / B5G command and control channel for some applications. Therefore, it is important to investigate how UAVs can be controlled via a $5 \mathrm{G}$ / B5G network and how they can improve the coverage of such networks by playing the role of flying radio base stations. All such investigations rely on the design of communication systems for UAVs.
According to [7] antenna techniques, radio propagation channels and multiple access mechanisms are among the most critical research issues in integrating UAVs into 5G networks. For all these issues, it is crucial to collect data on radio propagation in scenarios with UAVs. However, as discussed in [1] and [4], one needs expensive equipment to obtain real measurements using UAVs in millimeter waves (mmWaves, that correspond to frequencies higher than $20 \mathrm{GHz}$ ) for systems using multiple antennas and multipleinput multiple-output (MIMO) techniques. Hence, one important strategy is to use ray-tracing simulations to perform virtual field measurements and obtain data to design $5 \mathrm{G}$ communication systems for UAVs [7].

This context motivates this work, which describes the implementation of an ecosystem in which UAV simulators, wireless simulators, and network simulators are integrated, bringing a significant advance in obtaining propagation data from $5 \mathrm{G}$ simulations considering UVA scenarios. More specifically, this work proposes using a real-time 3D creation platform that interfaces with different simulators, which benefit from the realism of the 3D scenario. The Airsim framework is used for UVAs control. The other simulators are integrated via an API that communicates third-party software with the 3D scenario, as detailed in the next section.

\section{PROPOSED SOLUTION}

The proposed tool was designed as a software that uses different APIs to provide the desired features, such as the communication channel processing and the UAV flight simulator. The simulation process can be graphically represented by using the Unreal Game Engine (UE), which supports real-time event handling. Since it was made for gaming purposes, it is also suitable for user interaction.

To do network communication channel processing with the Unreal environment, a Python API developed by 20Tab's Team that establishes access of UE internal API by external scripts was adopted [6]. The necessity of external scripts exists due to the lack of native network communication solutions incorporated into the UE. Also, the use of the mentioned API creates the ability to check the network performance indicators using the game engine and test the validity and suitability of new telecommunication solutions like machine learning beam-selection [2]. 


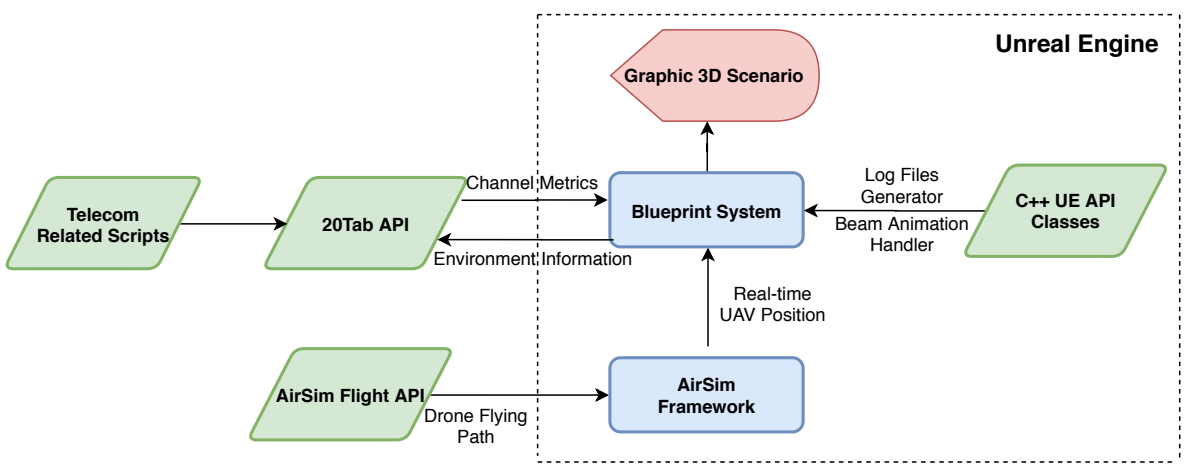

Figure 1: UAV Simulator integrated solution. Source: authors.

In our software, the UAV flight feature was implemented with AirSim, a high-fidelity visual and physical framework designed for simulations with cars and quadrotor drones [5]. The UAV's control can be done either using the framework's Python API or by connecting it with flight controllers that have MAVlink connection available. This enables automation in generating drone trajectories, which is important to collect data in realistic conditions for estimating 5G MIMO communication channels.

The development using UE is possible due to the blueprint system, a visual and node-based interface used for managing and dispatch actions depending on variables and events happening in the graphical environment. Each blueprint node is responsible for dispatching a specific action, and it is possible to create custom nodes with the game engine $\mathrm{C}++$ API. Therefore, custom nodes were built to implement several events related to the application, such as a simulation log file generator containing the desired parameters, graphic events related to the communication channels, retrieval of the drone's real-time position, and checking data validity.

Figure 1 displays the tools compounding the proposed solution and also describes how these technologies are related within the application design level.

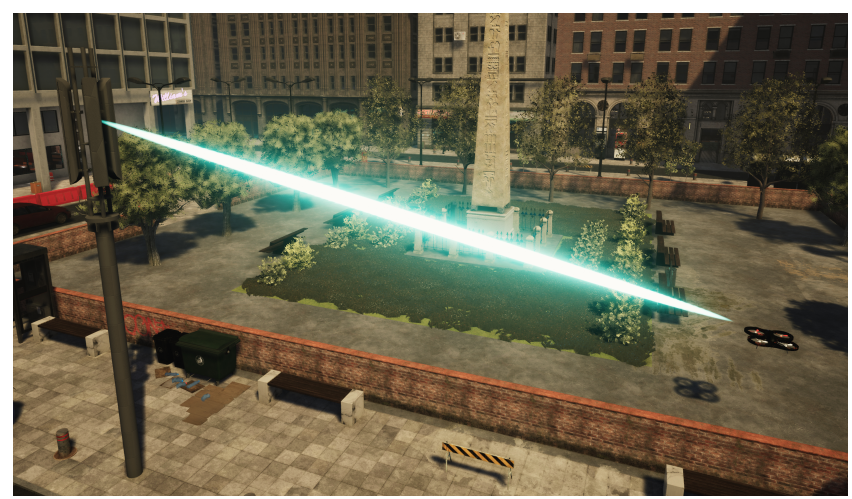

Figure 2: Antenna and drone communication granted by Unreal Engine. Source: authors.
Figure 2 illustrates the communication held between an antenna and a quadrotor (AirSim) in UE graphic scenarios. The blueprint system manages the antenna, which receives data from external inputs and checks if the connection is successful or not, so it can establish the mmWave beam animation.

During a simulation run, the estimator algorithm sends to Unreal Engine a vector with spherical coordinates of where the UAV will be located at that given instant of time, considering the antenna as the origin of the spherical coordinate system. The software receives the data held from the estimation script, and by using the real-time position of the drone, it verifies and warns the estimator if the communication was successfully held or not. The simulation also generates graphic events and registers the script attempts until the UAV end its trajectory, finishing the simulation and generating a log file with information concerning the success rate of the algorithm, the timestamp of the main events, and the drone relative position to the 5G Antenna, considering the Unreal Engine coordinate system.

Hence, the validity of the proposed solution can be verified by using both the graphic environment that behaves properly whenever one of the adopted third-parties software dispatches actions that change the context of the simulation, resulting in warnings or messages that appear during the simulation runs, as well as by analyzing the simulation log file, created for synthesizing all relevant pieces of information about the environment, in order to guarantee if it is working as expected.

\section{FINAL CONSIDERATIONS}

This work presented a new strategy for simulating $5 \mathrm{G}$ communication systems for UAVs.The performed tests out managed to connect the communication channel with a line of sight (LOS) using a geometric model. It intends to use more complex channel modeling techniques, like stochastic or Ray-tracing models, to cover NLOS situations in upcoming versions. It was also possible to extract a feedback from the UAV. This can be useful in studies about channel state information (CSI).

Future work includes developing a complete $5 \mathrm{G}$ telecommunications framework deeply integrated with the UE development kit. The proposed method brings a higher-level approach for setting 
up the simulation environment. It enables faster and easier customization of the scenario for $5 \mathrm{G}$ investigations in the academy and industry.

\section{REFERENCES}

[1] Felipe Bastos, Ailton Oliveira, João Borges, and Aldebaro Klautau. 2020. Effects of Environment Model Complexity in Ray-Tracing simulation for UAV Channels. $X$ Conferência Nacional em Comunicações, Redes e Segurança da Informação - ENCOM (2020).

[2] Aldebaro Klautau, Pedro Batista, Nuria Gonzalez-Prelcic, Yuyang Wang, and Robert W. Heath. 2018. 5G MIMO Data for Machine Learning: Application to BeamSelection Using Deep Learning. Information Theory and Applications Workshop (ITA) (2018)
[3] Mohammad Mozaffari, Ali Taleb Zadeh Kasgari, Walid Saad, Mehdi Bennis, and Mérouane Debbah. 2018. Beyond 5G with UAVs: Foundations of a 3D wireless cellular network. IEEE Transactions on Wireless Communications 18, 1 (2018), $357-372$.

[4] Ailton Oliveira, Marcus Dias, Isabela Trindade, and Aldebaro Klautau. 2019. RayTracing 5G Channels from Scenarios with Mobility Control of Vehicles and Pedestrians. XXXVII Simpósio Brasileiro de Telecomunicações e Processamento de SinaisSBrT (2019), 1-1.

[5] Shital Shah, Debadeepta Dey, Chris Lovett, and Ashish Kapoor. 2018. Airsim: High-fidelity visual and physical simulation for autonomous vehicles. In Field and service robotics. Springer, 621-635.

[6] 20tab srl. 2016. 20tab/UnrealEnginePython. https://github.com/20tab/ UnrealEnginePython

[7] Long Zhang, Hui Zhao, Shuai Hou, Zhen Zhao, Haitao Xu, Xiaobo Wu, Qiwu Wu, and Ronghui Zhang. 2019. A survey on $5 \mathrm{G}$ millimeter wave communications for UAV-assisted wireless networks. IEEE Access 7 (2019), 117460-117504. 